\title{
Investigation of vertical size segregation in bedload sediment transport with a coupled fluid-discrete element model
}

\author{
Philippe Frey ${ }^{1, *}$, Raphaël Maurin ${ }^{1,2}$, Lamia Morchid-Alaoui ${ }^{1}$, Shashank Gupta ${ }^{1}$, Julien Chauchat $^{3}$ \\ ${ }^{1}$ Université Grenoble Alpes, Irstea, UR ETNA, 2 rue de la Papeterie-BP 76, F-38402 St-Martin-d'Hères, France \\ ${ }^{2}$ Université de Toulouse, INPT, UPS, Institut de Mécanique des Fluides de Toulouse, Allée Camille Soula, 31400 Toulouse, France \\ ${ }^{3}$ Univ. Grenoble Alpes, LEGI, G-INP, CNRS, F-38000 Grenoble, France
}

\begin{abstract}
In order to gain understanding of kinetic sieving-type segregation in bedload sediment transport, numerical experiments of two-size particle mixtures were carried out, using a validated coupled fluiddiscrete element model developed at Irstea. A 3D 10\% steep domain consisting at initial time of a given number of layers of $4 \mathrm{~mm}$ particles deposited on top of a coarser $6 \mathrm{~mm}$ particle bed, was submitted to a turbulent and supercritical fluid shear flow (Shields numbers of 0.1 and 0.3 ). The elevation of the centre of mass of the infiltrated fine particles is observed to follow the same logarithmic decrease with time, whatever the initial number of fine layers. This decrease is steeper for a higher Shields number. The main result is that this typical behaviour is related at first order to the shear rate depth profile.
\end{abstract}

\section{Introduction}

Bedload transport by opposition to suspension is defined as the part of sediment transport remaining close to the bed in stream channels. It concerns the coarser grain material shear-driven by the turbulent water flow. It has major consequences for public safety, often aggravating flooding issues, for the management of water resources, and for environmental sustainability. Bedload transport investigations have mainly been focused on mass flux and its correlation with the water flow. Yet after more than a century of work we have no satisfactory theory for sediment transport. Empirical sediment transport formulas often poorly compare with field measurements, by sometimes order of magnitudes.

An important reason for our limited ability to predict sediment flux is the absence of general understanding of grain-grain interactions in stream channels [1,2].

Most particularly, in mountains, steep slopes drive an intense transport of a wide range of grain sizes leading to size segregation, also named grain size sorting [3]. This poorly understood phenomenon largely modifies fluxes and results in patterns that can be seen ubiquitously in nature.

Focusing on vertical size segregation, the infiltration of fine sediment into an immobile coarse bed has been extensively studied in fluvial geomorphology because of ecological considerations especially related to the reproduction of salmonids [4]. However when the coarse bed is moving, another type of infiltration named kinetic sieving can take place even with a coarse to fine diameter size ratio close to unity. Smaller grains may then move downward in the bed through voids that are opened by the general motion. It appears that this phenomenon which is important during floods has rarely been investigated in stream channels $[5,6]$.

In industrial processes, usually quite energetic, kinetic sieving-type segregation takes place, and has been more largely studied. Indeed, to be able to perfectly mix input materials and thus to counteract the natural segregation remains a challenge.

At Irstea, a coupled fluid-discrete element model has been developed and validated to study bedload transport [7]. In this paper we present a study of vertical size segregation and kinetic sieving using the model which has been extended to simulate two grain sizes. Following classical theories such as [8], segregation rates are analysed in term of shear rates.

\section{Coupled fluid-discrete element model}

A two-phase Eulerian-Lagrangian flow model developed at Irstea is used in this research. It is composed of a three dimensional discrete element model (DEM), describing each individual particle coupled with a one dimensional Reynolds average Navier Stokes (RANS) model [9].

The 3D DEM uses the open source code Yade [10] with a classical spring-dashpot contact law. The friction coefficient is set to 0.4 , and a restitution coefficient of 0.5 is taken consistently with the experimental validation [7].

In the RANS model, the water flow is considered to be stationary and unidirectional in the streamwise direction so that the fluid profile depends only on the depth (1D).

The momentum equation for the fluid is composed of the gravity term, the viscous stress, the turbulent

Corresponding author: philippe.frey@irstea.fr 
Reynolds stress and the fluid-particle interaction forces (restricted to drag and buoyancy). In our turbulent flow, the viscous stress is negligible. The turbulent stress is based on the eddy viscosity concept using an integral mixing length formulation, which allows recovering the law of the wall in clear fluid, while damping the turbulence down to zero at maximum packing fraction in the granular bed.

The drag coefficient depends on the local particle Reynolds number taking into account hindrance effects [9]. The fluid and particle velocity at the bottom of the domain and the fluid velocity gradient at the imposed free surface elevation are all set to zero.

The effect of the particles on the fluid phase is taken into account through the drag force and the solid volume fraction. Both the effect of the particle on the fluid and of the fluid on the particle are considered (two-way coupling). The model has been validated with turbulent bedload transport experiments, in terms of both the sediment transport rate and granular depth profiles. More details can be found in $[7,11]$. This model has also been used to analyse the dense granular rheology in turbulent bedload transport [12].

Our 3D numerical domain is made of a $10 \%\left(5.71^{\circ}\right)$ inclined channel filled with water, in which glass spherical particles (density of $2,500 \mathrm{~kg} / \mathrm{m}^{3}$ ) are deposited by gravity. At initial time, a given number $\mathrm{N}$ of layers of $4 \mathrm{~mm}$ diameter particles $(\mathrm{N}=0.05,0.5,1$ and 2) are deposited on top of a bed made of 7 layers of $6 \mathrm{~mm}$ diameter particles (Fig 1a). A layer is here defined as the number of particles necessary to occupy a volume corresponding to a depth of one diameter times the cell width and length, taking into account the maximum packing fraction.

The elevation of the water free-surface is imposed and creates a fluid flow driven by gravity on the top of the granular bed. The momentum input from gravity is balanced by the Reynolds shear stress in the dilute suspension layer and by the grain grain interactions in the sediment bed. The flow is turbulent (bulk Reynolds number of about $10^{4}$ ), hydraulically rough (particle Reynolds of order $10^{3}$ ) and supercritical (Froude greater than 1).

Both a low and a high free surface elevation have been set respectively at $10.3 d$ and $14 d$ where $d$ is the coarse diameter $(d=6 \mathrm{~mm})$, which yields respective Shields numbers $S h$ of 0.1 and 0.3 . The Shields number, classically used in sediment transport studies, is the dimensionless bottom fluid shear stress. It is defined as $S h=\frac{\tau_{b}}{(s-1) \cdot d}$ with $s$ the glass/water density ratio $(s=2.5) . \tau_{b}$ is unambiguously defined as the maximum of the fluid turbulent Reynolds shear stress.
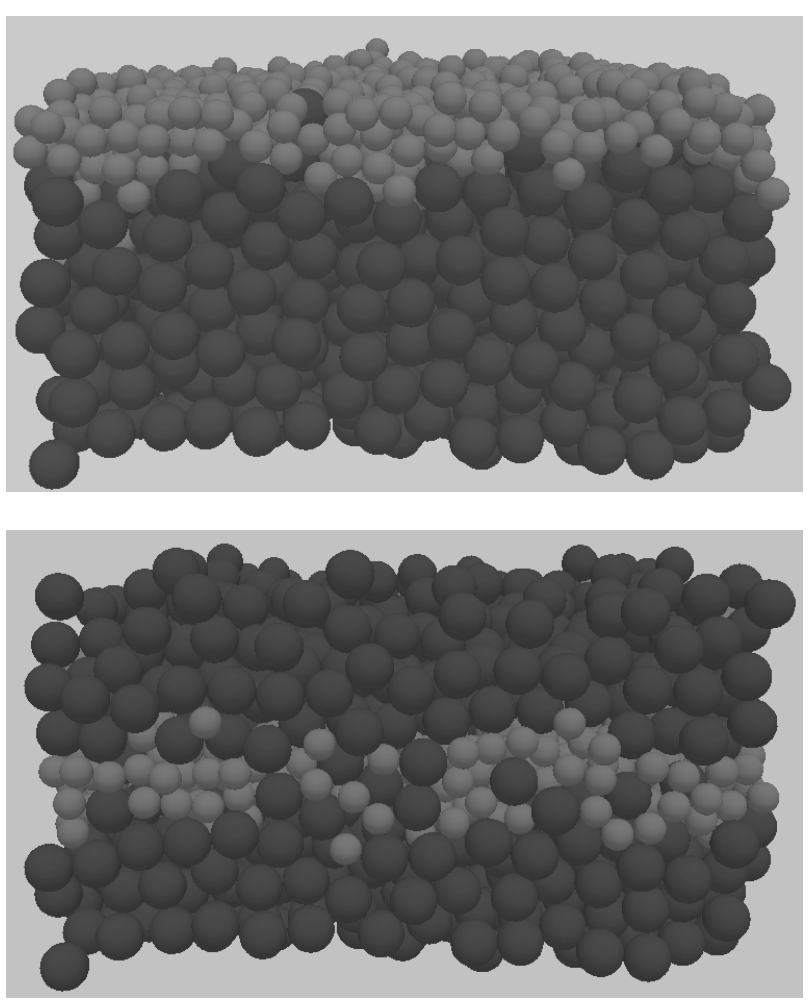

Fig. 1. Infiltration of two fine particle layers into the coarse bed a. $\mathrm{t}=0 \mathrm{~s}$ b. $\mathrm{t}=70 \mathrm{~s}(S h=0.3)$

Bi-periodic boundary conditions are imposed for the DEM, and only steady uniform conditions are analyzed. The unidirectional character of the fluid flow allows averaging spatially over the streamwise and spanwise directions, and analyzing the results only over the depth.

Due to kinetic sieving, the fine particles infiltrate through the coarser bed (Fig 1b). The centre of mass $z_{c}$ of the infiltrated particle cloud was calculated over time. To be able to follow the fine particles infiltrating in the lowermost coarser layers, it was necessary to simulate up to $10^{4}$ seconds ( 3 hours) for the $S h=0.1$ case.

\section{Results}

Both the fluid and particles starting from rest, the system follows a transient behaviour before reaching a characteristic steady state with a constant sediment transport rate.

Fig. 2 shows the evolution over time of the centre of mass of the fine particles $z_{c}$ for both Shields number of 0.1 and 0.3 (the origin of $z_{c}$ is at the bottom of the domain). The initial centre of mass position $z_{c}$ is dependent of the number $\mathrm{N}$ of fine particle layers since the number of coarse layers is kept constant and equal to 7 in the different simulations. 

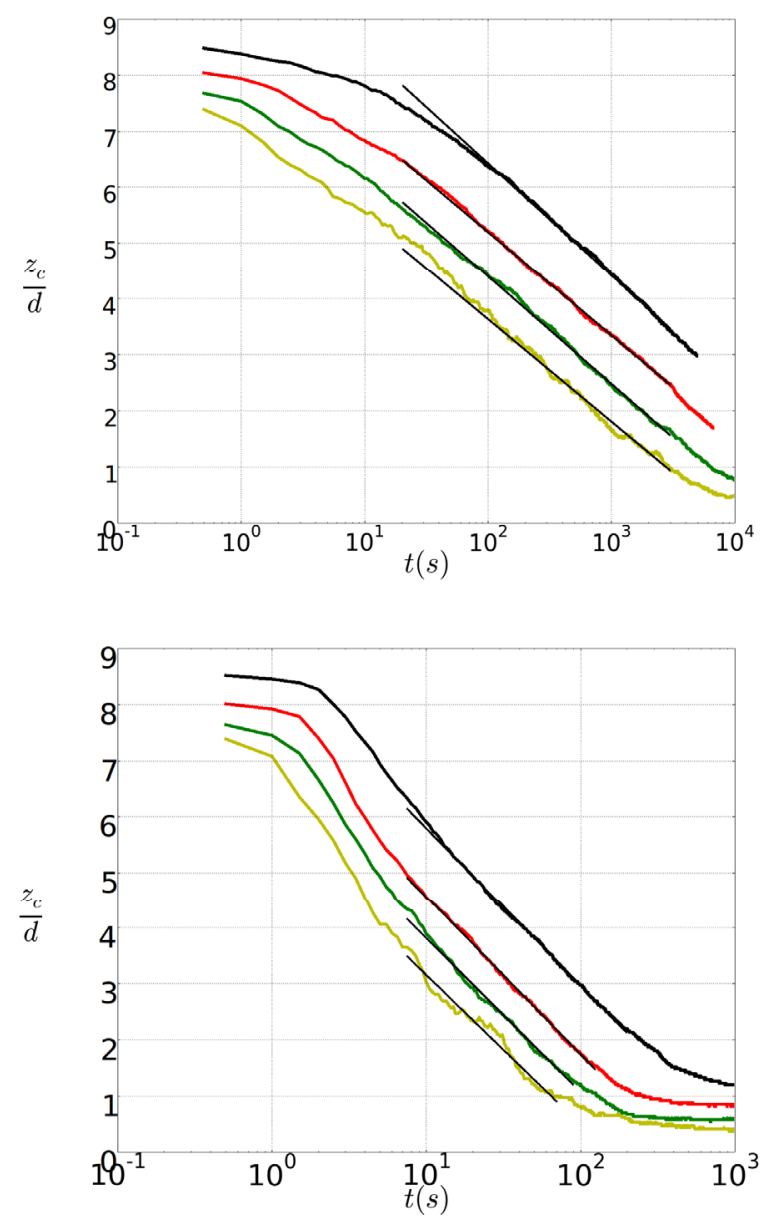

Fig. 2. Time evolution of the fine particles centre of mass for a number of layers $\mathrm{N}$ of $0.05,0.5,1$ and 2 , from light yellow $(\mathrm{N}=0.05$ bottom) to dark black ( $\mathrm{N}=2$ top). See text for the slope of the fit a. Shields of $0.1 \mathbf{b}$. Shields of 0.3 .

As one would expect, the infiltration evolution depends on the Shields number. For $S h=0.3$, the cloud of fine particles reached the bottom of the domain between $10^{2}$ and $10^{3} \mathrm{~s}$, whereas this duration was above $10^{4} \mathrm{~s}$ for the $S h=0.1$ case.

Considering the transient behaviour, both the number of fine particle layer deposited $\mathrm{N}$ and the Shields number influence the evolution of the fine particles centre of mass (Fig. 2). During this period, the size-segregation phenomenon is coupled with the evolution of the system to the steady state, making a clear analysis complicated.

Therefore, we rather focus on the steady state, which corresponds to the infiltration of fine particles through the coarse granular bed in quasi-static motion. In this region, the fine particles behaviour does not depend any more on the number $\mathrm{N}$ (provided the cloud is not yet affected by the bottom) and their centre of mass position $z_{c}$ decreases linearly with $\log (t)$ (Fig.2).

This linear trend was fitted on all curves (Fig.2). For a given Shields number, the slope was found to be the same. For $S h=0.1$ (Fig. 2a), the mean slope (out of the four $\mathrm{N}$ cases) amounts to -0.83 (min of -0.84 and max of -0.80 ). For $S h=0.3$ (Fig. 2b), the mean is -1.19 ( $\mathrm{min}$ of 1.22 and max of -1.16 ). Surprisingly the segregation rate does not depend of the concentration of fines, even for $\mathrm{N}=0.05$ corresponding to a low number of fine particles initially scattered on top of the coarse bed, though the evolution is a bit less smooth than for higher N. As could be expected, the segregation rate (given by the absolute slope) increases with the Shields number.

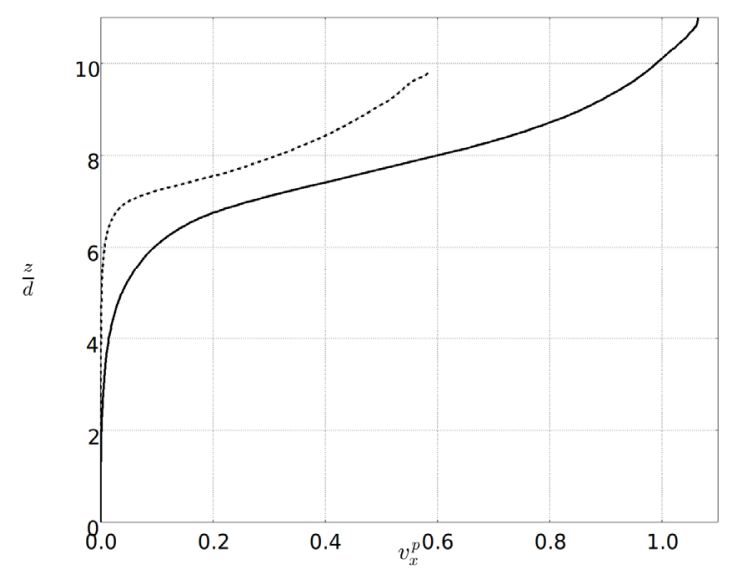

Fig. 3. Depth profiles of the streamwise particle velocity $(\mathrm{m} / \mathrm{s})$ for both Shields numbers of 0.1 (dashed) and 0.3 (plain).

Following theories such as Savage and Lun [8] (see also a simplified description in [13] ), we will consider herein that the shear rate plays the major role. the segregation rate is taken proportional to the shear rate $\frac{d z_{c}}{d t} \propto-\dot{\gamma}$. Streamwise coarse-only particle velocity depth profiles appear in Fig. 3 for both Shields numbers. An exponential decrease is observed in the quasi-static part of the bed [11] as evidenced on Fig. 3 and more precisely on Fig. 4 (semi-logarithmic): $v^{p}{ }_{x} \propto e^{z / a}$. This means that the shear rate $\dot{\gamma}=\frac{d v^{p} x}{d z}$ also decreases exponentially. As a result, the segregation rate $\frac{d z_{c}}{d t} \propto-e^{z / a}$. Separating variables and integrating from time $t=0$ and initial position gives $z_{c}=-a \log (t / a+b)$ with $\mathrm{b}$ an integration constant. When $t>>1$, $z_{c}=-a \log (t)+c$.

According to this simple calculation, the fitted slope $a$ appearing on Fig. 2 should be the same as that of the exponential decay of the particle velocity profile. Velocity profiles were fitted over the exponential domain in Fig. $4 \mathrm{a}(S h=0.1)$ and Fig. $4 \mathrm{~b}(S h=0.3)$ yielding mean values of respectively 0.80 ( $\min$ of 0.76 and $\max$ of 0.86 ) and 1.07 ( $\mathrm{min}$ of 1.01, $\max$ of 1.12). Those values compare very well with the segregation evolution values (Fig. 2). For $S h=0.1$, the difference of slopes is only $4 \%$ while it amounts to $11 \%$ for $S h=0.3$. 

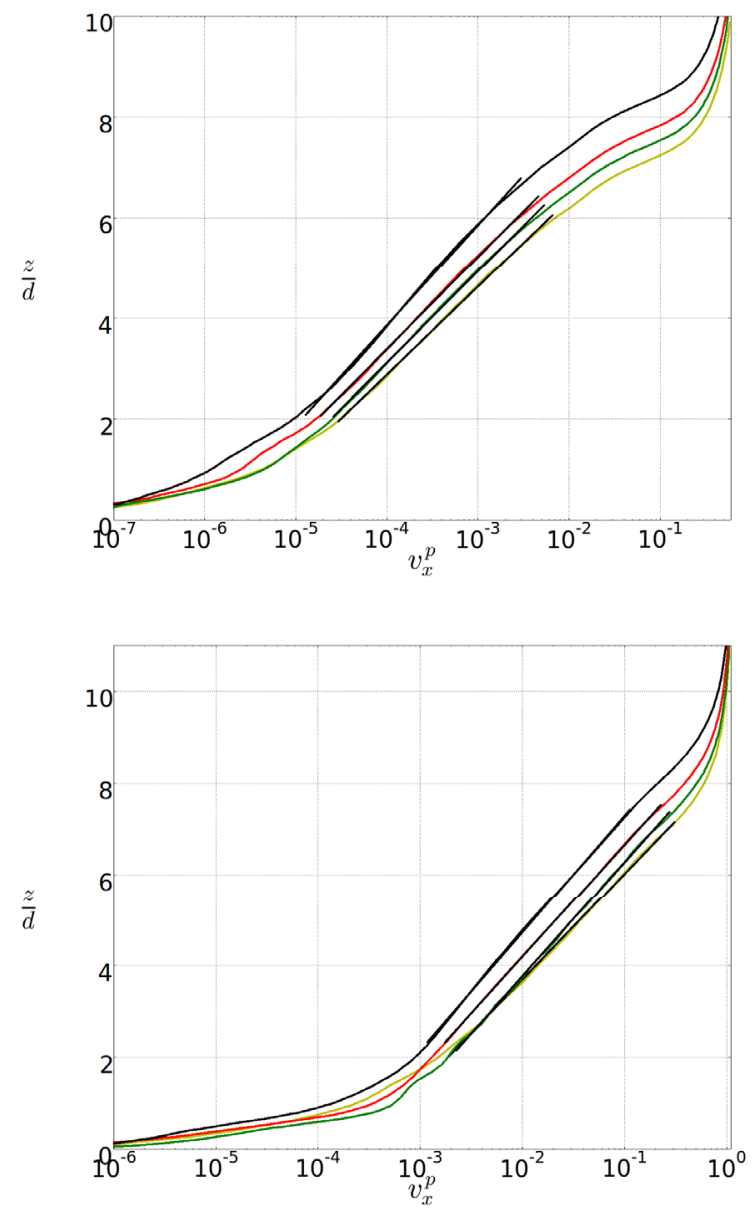

Fig. 4. Depth profiles of the streamwise particle velocity $(\mathrm{m} / \mathrm{s})$ with fitting in the exponential domain (see text for fit values) from light yellow ( $\mathrm{N}=0.05$ bottom) to dark black ( $\mathrm{N}=2$ top). $\mathbf{a}$. Shields of $0.1 \mathbf{b}$. Shields of 0.3 .

\section{Conclusion}

After the initial transient period, the elevation of the centre of mass of the infiltrated fine particles is observed to follow the same logarithmic decrease with time, whatever the initial number of fine layers, from a few scattered fine particles up to two full fine layers. This decrease is steeper for a higher Shields number.

The main finding is that this typical behaviour is related at first order to the shear rate exponential depth profile present in the quasi-static region. These results confirm that the shear rate is the main driver of the kinetic sieving-type observed segregation in our bedload experiments.

While this is consistent with established theoretical framework for the largest number of fine particle layers, one would expect a different type of behaviour for isolated particles. Indeed an asymmetry between fine particles surrounded by large particles vs. large particles surrounded by fine particles has been reported [14]. Future work will therefore be devoted to the study of single fine particle behaviour in a similar system.
A better understanding of size segregation poorly known processes will ultimately improve sediment transport and river morphology modelling.

This research was supported by Irstea (formerly Cemagref), the labex OSUG@2020, the French Institut National des Sciences de l'Univers programs EC2CO-BIOHEFECT and EC2COLEFE MODSED, and by ANR (the French national research agency) project ANR-16-CE01-0005.

\section{References}

1 P. Frey and M. Church, Science 325, 1509 (2009).

2 P. Frey and M. Church, Earth Surface Processes and Landforms 36, 58 (2011).

3 V. Hergault, P. Frey, F. Métivier, C. Barat, C. Ducottet, T. Böhm, and C. Ancey, Experiments in Fluids 49, 1095 (2010).

4 A. R. Dudill, P. Frey, and M. Church, Earth Surface Processes and Landforms, DOI: 10.1002/esp.4080 (in press).

5 V. Bacchi, A. Recking, N. Eckert, P. Frey, G. Piton, and M. Naaim, Earth Surface Processes and Landforms 39, 1075 (2014).

6 A. Recking, P. Frey, A. Paquier, and P. Belleudy, Journal of Geophysical ResearchEarth Surface 114, F03010 (2009).

7 R. Maurin, J. Chauchat, B. Chareyre, and P. Frey, Phys. Fluids 27, 113302 (2015).

8 S. B. Savage and C. K. K. Lun, J. Fluid Mech. 189, 311 (1988).

9 T. Revil-Baudard and J. Chauchat, J. Geophys. Res.-Oceans 118, 619 (2013).

10 Śmilauer et al., Yade Documentation 2nd ed. The Yade Project. DOI 10.5281/zenodo.34073 (http://yade-dem.org/doc/), 2015).

11 P. Frey, Earth Surface Processes and Landforms 39, 646 (2014).

12 R. Maurin, J. Chauchat, and P. Frey, J. Fluid Mech. 804, 490 (2016).

13 B. Andreotti, Y. Forterre, and O. Pouliquen, Granular Media: Between Fluid and Solid (Cambridge University Press, 2013).

14 K. van der Vaart, P. Gajjar, G. Epely-Chauvin, N. Andreini, J. Gray, and C. Ancey, Phys. Rev. Lett. 114, 10.1103 (2015). 